\title{
Coronary arteriography
}

\author{
Richard Emanuel ${ }^{1}$
}

In December 1973 a letter from one of Her Majesty's coroners was put before the Cardiology Committee of the Royal College of Physicians asking for current views on coronary arteriography in the U.K. and an estimate of the risks involved.

The committee discussed this letter on the basis that coronary arteriography was an established technique, and irrespective of the future of coronary artery surgery it had achieved a permanent place in the investigation of cardiac patients.

They appreciated from the published data that the hazards included sudden death and that the mortality might be as high as 5 per cent in centres where the technique was being established. In experienced hands this figure fell to the more acceptable level of 0.3 per cent and in the most experienced centres a mortality rate of 0.1 per cent might be achieved (Adams, Fraser, and Abrams, 1973). It was also appreciated there was a significant morbidity which included myocardial infarction, systemic embolism, and arterial damage, but it was evident that mortality, the complication rate, and the duration of the procedure were inversely related to the expertise of the operators, though the severity of the disease and the clinical condition of individual patients studied were also factors (Judkins and Gander, 1974).

During the discussions it became apparent that there was little information available on coronary arteriography in the U.K. and it was agreed that the present position should be investigated. It was decided that information should be obtained by questionnaire, though the shortcomings of this mode of data collection were appreciated. Doubt was expressed about the quality of the data which would be available and as the survey proceeded it became clear that these apprehensions were fully justified.

Eighty cardiologists, radiologists, and physicians

${ }^{1}$ Secretary of the Standing Committee on Cardiology, Royal College of Physicians, London. with a special interest in cardiology were sent a preliminary questionnaire. All replied and it was evident that in 1973 coronary arteriography was being carried out in 50 centres in England, Scotland, Wales, Northern Ireland, and Eire. In the same year it was estimated that between 3000 and 3500 coronary arteriograms had been done, but in only 8 centres, 7 of which were in London, were more than IOO investigations being carried out per annum. In 17 centres between 50 and 100 investigations were done each year and in the remaining 25 centres fewer than 50 investigations were undertaken per annum. In II of the 50 centres $(22 \%)$ there were no facilities for cardiac surgery within the same building or building complex where the coronary arteriography was being performed.

A second questionnaire was circulated to find out the mortality and morbidity. Replies were received from 46 of the 50 centres and the overall figures were disturbing. Only 9 of the 50 centres were able to specify the exact mortality and morbidity and relate it to the number of investigations per annum. At only one of these centres was there a zero mortality. At the remaining 8 the mortality was not less than 0.6 per cent and the morbidity varied from 0.9 to 2.2 per cent. A breakdown of these figures will be given to the British Cardiac Society. A further disquieting fact was the inadequacy of the records available.

The committee examined these data and considered that, in view of the risks, this investigation should be carried out only in a limited number of centres where there were skilled personnel, fully trained in the technique, who were performing coronary arteriography regularly.

It was accepted that the majority of investigations would be in patients with coronary artery disease who were being considered for myocardial revascularization. Other indications included contemplated valve replacement, particularly in older 
patients who might have additional coronary artery disease, and the investigation of a limited number of cases with atypical chest pain, also those with suspected congenital or other unusual coronary artery lesions. There was also an increasing place for coronary arteriography in some patients with unstable angina (coronary insufficiency). The investigation in these cases could be particularly hazardous as it might provoke severe prolonged pain. However, it was likely that despite the possibility of increased risks in this situation, coronary artery surgery could be life saving.

The committee put forward the following recommendations which they thought might be helpful, particularly to those who were considering starting coronary arteriography.

I) Coronary arteriography should be carried out only in centres where right and left heart catheterization were routine procedures. This inferred that there would be a cardiologist or physician with special experience in cardiology available.

2) The problem of personnel was difficult but in principle there should be a minimum number of operators who actually inserted coronary artery catheters. Ideally, there should be one consultant cardiologist, or consultant radiologist supported by a cardiologist and one junior hospital doctor, probably a senior registrar who was being trained in the technique.

3) As in all procedures with potential risks, there was a minimum number of investigations below which these hazards increased. The committee considered that ideally this figure should not fall below too per year at any centre.

4) To minimize the risk at centres starting coronary arteriography the committee suggested that the senior man involved (presumably a consultant cardiologist or senior registrar in cardiology) should be seconded for a minimum period of three months to a centre where coronary arteriography was part of the daily programme. The trainee should already be well versed in cardiac catheterization so that he could acquire the necessary expertise in coronary arteriography with minimal delay. During this training period he should be an active member of the team and not simply an observer.

5) In all new centres facilities for emergency cardiac surgery should be planned within the same building or building complex, and when cases of above average risk were being investigated the theatre should be accessible, with a surgeon alerted. In centres where coronary arteriography is already successfully established but in which there is no theatre adjacent to the haemodynamic laboratory, adequate alternative arrangements for emergency surgery are acceptable.

6) The committee suggested that it would be unwise for a new centre to start coronary arteriography unless it could be envisaged that in the foreseeable future demands would not fall below and would eventually exceed 100 investigations a year. 7) Finally, the usual radiation protection monitoring procedures to deal with radiation hazards should be adopted in a similar way to those recommended for all other investigations involving cardiac catheterization and angiography.

\section{References}

Adams, D. F., Fraser, D. B., and Abrams, H. L. (1973). The complications of coronary arteriography. Circulation, 48, 609.

Judkins, M. P., and Gander, M. P. (1974). Prevention of complications of coronary arteriography. Circulation, 49, 599.

Requests for reprints to Dr. Richard Emanuel, 6 Upper Wimpole Street, London WIM 7TD. 